Pacific Journal of Mathematics

ructions 


\title{
A LATTICE-THEORETICAL FIXPOINT THEOREM AND ITS APPLICATIONS
}

\author{
ALFRED TARSKI
}

1. A lattice-theoretical fixpoint theorem. In this section we formulate and prove an elementary fixpoint theorem which holds in arbitrary complete lattices. In the following sections we give various applications (and extensions) of this result in the theories of simply ordered sets, real functions, Boolean algebras, as well as in general set theory and topology. ${ }^{1}$

By a lattice we understand as usual a system $\mathfrak{U}=\langle A, \leq\rangle$ formed by a nonempty set $A$ and a binary relation $\leq$; it is assumed that $\leq$ establishes a partial order in $A$ and that for any two elements $a, b \in A$ there is a least upper bound (join) $a \cup b$ and a greatest lower bound (meet) $a n b$. The relations $\geq$, <, and $>$ are defined in the usual way in terms of $\leq$.

The lattice $\mathfrak{Q}=\langle A, \leq\rangle$ is called complete if every subset $B$ of $A$ has a least upper bound $U B$ and a greatest lower bound $\cap B$. Such a lattice has in particular two elements 0 and 1 defined by the formulas

$$
0=\cap A \text { and } 1=\bigcup A
$$

Given any two elements $a, b \in A$ with $a \leq b$, we denote by $[a, b]$ the interval with the endpoints $a$ and $b$, that is, the set of all elements $x \in A$ for which $a \leq x \leq b$; in symbols,

$$
[a, b]=E_{x}[x \in A \text { and } a \leq x \leq b]
$$

The system $\langle[a, b], \leq\rangle$ is clearly a lattice; it is a complete if $\mathscr{O}$ is complete.

We shall consider functions on $A$ to $A$ and, more generally, on a subset $B$ of $A$ to another subset $C$ of $A$. Such a function $f$ is called increasing if, for any

1 For notions and facts concerning lattices, simply ordered systems, and Boolean algebras consult $[\mathbf{1}]$.

Received June 29, 1953. Most of the results contained in this paper were obtained in 1939. A summary of the results was given in [6]. The paper was prepared for publication when the author was working on a research project in the foundations of mathematics spons ored by the Office of Ordnance Research, U.S. Army.

Pacific J. Math. 5 (1955), 285-309 
elements $x, y \in B, x \leq y$ implies $f(x) \leq f(y)$. By a fixpoint of a function $f$ we understand, of course, an element $x$ of the domain of $f$ such that $f(x)=x$.

Throughout the discussion the variables $a, b, \cdots, x, y, \cdots$ are assumed to represent arbitrary elements of a lattice (or another algebraic system involved).

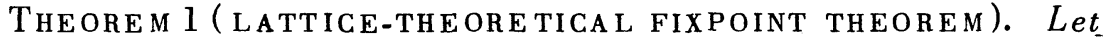

(i) $\mathfrak{U}=\langle A, \leq\rangle$ be a complete lattice,

(ii) $f$ be an increasing function on $A$ to $A$,

(iii) $P$ be the set of all fixpoints of $f$.

Then the set $P$ is not empty and the system $\langle P, \leq\rangle$ is a complete lattice; in particular we have

$$
U P=U E_{x}[f(x) \geq x] \in P
$$

and

$$
\cap P=\cap E_{x}[f(x) \leq x] \in P .^{2}
$$

Proof. Let

$$
u=U E_{x}[f(x) \geq x]
$$

We clearly have $x \leq u$ for every element $x$ with $f(x) \geq x$; hence, the function $f$ being increasing,

$$
f(x) \leq f(u) \text { and } x \leq f(u)
$$

By (1) we conclude that

$$
u \leq f(u) \text {. }
$$

2 In 1927 Knaster and the author proved a set theoretical fixpoint theorem by which every function, on and to the family of all subsets of a set, which is increasing under set-theoretical inclusion has at least one fixpoint; see [3], where some applications of this result in set theory (a generalization of the Cantor-Bernstein theorem) and topology are also mentioned. A generalization of this result is the lattice-theoretical fixpoint theorem stated above as Theorem 1 . The theorem in its present form and its various applications and extensions were found by the author in 1939 and discussed by him in a few public lectures in 1939-1942. (See, for example, a reference in the American Mathematical Monthly 49(1942), 402. ) An essential part of Theorem 1 was included in $[1$, p. 54]; however, the author was informed by Professor Garrett Birkhoff that a proper historical reference to this result was omitted by mistake. 
Therefore

$$
f(u) \leq f(f(u))
$$

so that $f(u)$ belongs to the set $\mathrm{E}_{x}[f(x) \geq x]$; consequently, by (1),

$$
f(u) \leq u
$$

Formulas (2) and (3) imply that $u$ is a fixpoint of $f$; hence we conclude by (1) that $u$ is the join of all fixpoints of $f$, so that

$$
U P=U_{x}[f(x) \geq x] \in P .
$$

Consider the dual lattice $\mathfrak{U}^{\prime}=\langle A, \geq\rangle$. $\mathfrak{U}$, like $\mathfrak{U}$, is complete, and $f$ is again an increasing function in $\mathfrak{U}$ '. The join of any elements in $\mathfrak{C}^{\prime}$ obviously coincides with the meet of these elements in $\mathscr{X}$. Hence, by applying to $\mathscr{C U}^{\prime}$ the result established for $\mathscr{U}$ in (4), we conclude that

$$
\cap P=\cap \mathrm{E}_{x}[f(x) \leq x] \in P
$$

Now let $Y$ be any subset of $P$. The system

$$
\mathfrak{B}=\langle[\cup Y, 1], \leq\rangle
$$

is a complete lattice. For any $x \in Y$ we have $x \leq U Y$ and hence

$$
x=f(x) \leq f(U Y)
$$

therefore $U Y \leq f(\cup Y)$. Consequently, $U Y \leq z$ implies

$$
U Y \leq f(U Y) \leq f(z)
$$

Thus, by restricting the domain of $f$ to the interval $[U Y, 1]$, we obtain an increasing function $f^{\prime}$ on $[U Y, 1]$ to $[U Y, 1]$. By applying formula (5) established above to the lattice $B$ and to the function $f^{\prime}$, we conclude that the greatest lower bound $v$ of all fixpoints of $f^{\prime}$ is itself a fixpoint of $f^{\prime}$. Obviously, $v$ is a fixpoint of $f$, and in fact the least fixpoint of $f$ which is an upper bound of all elements of $Y$; in other words, $v$ is the least upper bound of $Y$ in the system $\langle P, \leq\rangle$. Hence, by passing to the dual lattices $\mathscr{U}^{\prime}$ and $\mathfrak{B}^{\prime}$, we see that there exists also a greatest lower bound of $Y$ in $[P, \leq]$. Since $Y$ is an arbitrary subset of $P$, we finally conclude that

the system $\langle P, \leq\rangle$ is a complete lattice. 
In view of (4) -(6), the proof has been completed.

By the theorem just proved, the existence of a fixpoint for every increasing function is a necessary condition for the completeness of a lattice. The question naturally arises whether this condition is also sufficient. It has been shown that the answer to this question is affirmative. ${ }^{3}$

A set $F$ of functions is called commutative if

(i) all the functions of $F$ have a common domain, say $B$, and the ranges of all functions of $F$ are subsets of $B$;

(ii) for any $f_{3} g \in F$ we have $f g=g f$, that is,

$$
f(g(x))=g(f(x)) \text { for every } x \in B \text {. }
$$

Using this notion we can improve Theorem 1 in the following way:

THEOREM 2 (GENERALIZED LATTICE-THEORETRICAL FIXPOINT THEO$\mathrm{REM})$. Let

(i) $\mathscr{Q}=\langle A, \leq\rangle$ be a complete lattice,

(ii) F be any commutative set of increasing functions on $A$ to $A$,

(iii) $P$ be the set of all common fixpoints of all the functions $f \in F$.

Then the set $P$ is not empty and the system $\langle P, \leq\rangle$ is a complete lattice; in particular, we have

$$
U P=U E_{x}[f(x) \geq x \text { for every } f \in F] \in P
$$

and

$$
\cap P=\cap \mathrm{E}_{x}[f(x) \leq x \text { for every } f \in F] \in P .
$$

Proof. Let

$$
u=U E_{x}[f(x) \geq x \text { for every } f \in F]
$$

As in the proof of Theorem 1 we show that

$$
u \leq f(u) \text { for every } f \in F .
$$

Given any function $g \in F$, we have, by (2),

3 This is a result of Anne C. Davis; see her note [2] immediately following this this paper. 


$$
g(u) \leq g(f(u))
$$

and hence, the set $F$ being commutative,

$$
g(u) \leq f(g(u))
$$

for every $f \in F$. Thus

$$
g(u) \in \mathbb{E}_{x}[f(x) \geq x \text { for every } f \in F]
$$

Therefore, by (1),

$$
g(u) \leq u
$$

since $g$ is an arbitrary function of $F$, we have

$$
f(u) \leq u \text { for every } f \in F .
$$

From (1)-(3) we conclude that $u$ is a common fixpoint of all functions $f \in F$, and, in fact, the least upper bound of all such common fixpoints. In other words,

$$
\cup P=U_{x}[f(x) \geq x \text { for every } f \in F] \in P
$$

In its remaining part the proof is entirely analogous to that of Theorem 1.

Since every set consisting of a single function is obviously commutative, Theorem 2 comprehends Theorem 1 as a particular case. Theorem 2 will not be involved in our further discussion.

2. Applications and extensions in the theories of simply ordered sets and real functions. A simply ordered system $\mathscr{U}=\langle A, \leq\rangle$, that is, a system formed by a nonempty set $A$ and a binary relation $\leq$ which establishes a simple order in $A$, is obviously a lattice. If it is a complete lattice, it is called a continuously (or completely) ordered system. The system $\mathcal{U}$ is said to be a densely ordered system if, for all $x_{\imath} y \in A$ with $^{-}<y$, there is a $z \in A$ with $x<z<y$.

Theorems 1 and 2 obviously apply to every continuously ordered system \&ै. Under the additional assumption that $\mathcal{U}$ is densely ordered we can improve Theorem 1 by introducing the notions of quasi-increasing and quasi-decreasing functions.

Given a function $f$ and a subset $X$ of its domain, we denote by $f^{*}(X)$ the set of all elements $f(x)$ correlated with elements $x \in X$. A function $f$ on $B$ to $C$, where $B$ and $C$ are any two subsets of $A$, is called quasi-increasing if it satisfies the formulas 


$$
f(\mathrm{U} X) \geq \cap f^{*}(X) \text { and } f(\cap X) \leq \mathrm{U} f^{*}(X)
$$

for every nonempty subset $X$ of $B$. It is called quasi-decreasing if it satisfies the formulas

$$
f(U X) \leq \cup f^{*}(X) \text { and } f(\cap X) \geq \cap f^{*}(X)
$$

for every nonempty subset $X$ of $A$. A function which is both quasi-increasing and quasi-decreasing is called continuous.

\section{THEOREM 3. Let}

(i) $\mathscr{U}=\langle A, \leq\rangle$ be a continuously and densely ordered set,

(ii) $f$ be a quasi-increasing function and $g$ a quasi-decreasing function on $A$ to $A$ such that

$$
f(0) \geq g(0) \text { and } f(1) \leq g(1),
$$

(iii) $P=E_{x}[f(x)=g(x)]$.

Then $P$ is not empty and $\langle P, \leq\rangle$ is a continuously ordered system; in particular we have

$$
U P=U E_{x}[f(x) \geq g(x)] \in P
$$

and

$$
\cap P=\cap E_{x}[f(x) \leq g(x)] \in P .
$$

Proof. Let $B$ be any subset of $A$ such that

$$
f(x) \geq g(x) \text { for } x \in B
$$

Assume that

$$
f(U B)<g(U B) .
$$

Since, by hypothesis, $f(0) \geq g(0)$, we conclude that

$$
\mathrm{U} B \neq 0 \text {. }
$$

The system 2 being densely ordered, we also conclude from (2) that there is an element $a \in A$ for which 


$$
f(\cup B)<a<g(\cup B) .
$$

Let

$$
D=E_{x}[x \leq \cup B \text { and } g(x) \leq a]
$$

whence

$$
\mathrm{U} D \leq \mathrm{U} B
$$

and

$$
U_{g^{*}}(D) \leq a .
$$

If $U D=\cup B$, we see from (3) that $U D \neq 0$ and that consequently the set $D$ is not empty; hence, the function $g$ being by hypothesis quasi-decreasing, we obtain

$$
g(U B)=g(\cup D) \leq U_{g} *(D),
$$

and therefore, by ( 7),

$$
g(\cup B) \leq a .
$$

Since this formula clearly contradicts (4) we conclude that $U D \neq \cup B$ and thus, by (6),

$$
\mathrm{U} D<\mathrm{U} B
$$

Let

$$
E=\mathrm{E}_{x}[\cup D<x \text { and } x \in B] \text {. }
$$

If the set $E$ were empty, we would have $x \leq \cup D$ for every $x \in B$ and consequently $\cup B \leq \cup D$, in contradiction to (8). Hence $E$ is not empty. We easily conclude by $(9)$ that $\cup E=\cup B$. Since, by hypothesis, the function $f$ is quasiincreasing, we have

$$
f(U B)=f(U E) \geq \cap f^{*}(E)
$$

and therefore, by (4),

$$
a>\cap f^{*}\left(E^{\prime}\right) .
$$

Hence we must have $a>f(z)$ for some $z \in E$, for otherwise 


$$
a \leq \cap f^{*}(E)
$$

Thus, by (1) and (9),

$$
\cup D<z, z \in B \text {, and } g(z) \leq a \text {; }
$$

therefore, by (5), $z \in D$. The formulas

$$
\cup D<z \text { and } z \in D
$$

clearly contradict each other.

We have thus shown that formula (2) cannot hold for any non-empty set $B$ satisfying (1). In other words, we have

$$
\begin{gathered}
f(U B) \geq g(U B) \text { for every non-empty subset } B \text { of } \\
E_{x}[f(x) \geq g(x)] .
\end{gathered}
$$

By applying the result just obtained to the dual system $\mathfrak{O}^{\circ}=\left\langle A_{z} \geq\right\rangle$, we conclude that

$$
\begin{gathered}
f(\cap C) \leq g(\cap C) \text { for every subset } C \text { of } \\
E_{x}[f(x) \leq g(x)] .
\end{gathered}
$$

Now let $Y$ be any subset (whether empty or not) of the set

$$
P=\mathrm{E}_{x}[f(x)=g(x)] \text {, }
$$

and let

$$
u=U E_{x}[f(x) \geq g(x) \text { and } x \leq \cap Y]
$$

By (10) and (11) we have

$$
f(u) \geq g(u) \text { and } f(\cap Y) \leq g(\cap Y) \text {. }
$$

Hence, in case $u=\cap Y$, we obtain at once

$$
f(u)=g(u) \text {, that is, } u \in P \text {. }
$$

In case $u \neq \cap Y$ we see from (12) that $u<\cap Y$. The system \& being densely ordered, we conclude that 


$$
u=\cap E_{x}[u<x \leq \cap Y] .
$$

We also see from (12) that $f(x)<g(x)$ for every element $x$ of the set

$$
E_{x}[u<x \leq \cap Y] \text {. }
$$

Hence, by (11) and (15), we obtain

$$
f(u) \leq g(u),
$$

and this formula, together with (13), implies (14) again. Thus we have shown that

(16) for every subset $Y$ of $P$, if $u=\mathrm{UE}_{x}[f(x) \geq g(x)$ and $x \leq \mathrm{UY}]$, then $u \in P$.

Dually we have

(17) for every subset $Y$ of $P$, if $v=\cap \mathrm{E}_{x}[f(x \leq g(x)$ and $x \geq \cap Y]$, then $v \in P$.

We see immediately that the element $u$ in $(16)$ is the largest element of $P$ which is a lower bound of all elements of $Y$; in other words, $u$ is the greatest lower bound of $Y$ in the system $\langle P, \leq\rangle$. Similarly, the element $v$ in $(17)$ is the least upper bound of $Y$ in $\langle P, \leq\rangle$. Consequently,

$$
\langle P, \leq\rangle \text { is a continuously ordered system. }
$$

Finally, let us take in (16) and (17) the empty set for $Y$, so that $\cap Y=1$ and $U Y=0$. We then easily arrive at formulas

$$
U P=\mathrm{UE}_{x}[f(x) \geq g(x)] \in P
$$

and

$$
\cap P=\cap E_{x}[f(x) \leq g(x)] \in P .
$$

By (18)-(20) the proof is complete.

Every increasing function is clearly quasi-increasing. The identity function, $g(x)=x$ for every $x \in A$, is continuous, that is, both quasi-increasing and quasi-decreasing, and the same applies to every constant function, $g(x)=c \in A$ for every $x \in A$. Hence we can take in Theorem 3 an arbitrary increasing function 
for $f$ and the identity function for $g$; we thus obtain Theorem 1 in its application to continuously and densely ordered systems. On the other hand, by taking for $g$ a constant function, we arrive at:

Theorem 4 (Generalized Weierstrass theorem). Let

(i) $\mathscr{A}=\langle A, \leq\rangle$ be a continuously and densely ordered system,

(ii) $f$ be a quasi-increasing function on $A$ to $A$ and $c$ be an element of $A$ such that

$$
f(0) \geq c \geq f(1)
$$

(iii) $P=\mathrm{E}_{x}[f(x)=c]$.

Then $P$ is not empty and $\langle P, \leq\rangle$ is a continuously ordered system; in particular, we have

$$
U P=U E_{x}[f(x) \geq c] \in P
$$

and

$$
\cap P=\cap \mathrm{E}_{x}[f(x) \leq c] \in P .
$$

An analogous theorem for pseudo-decreasing functions can be derived from Theorem 3 by taking an arbitrary constant function for $f$.

It can be shown by means of simple examples that Theorems 3 and 4 do not extend either to arbitrary continuously ordered systems or to arbitrary complete lattices which satisfy the density condition (that is, in which, for any elements $x$ and $y, x<y$ implies the existence of an element $z$ with $x<z<y$ ).

We can generalize Theorem 3 by considering two simply ordered systems,

$$
\mathfrak{U}=\langle A, \leq\rangle \text { and } B=\langle B, \leq\rangle \text {, }
$$

as well as two functions on $A$ to $B$, a quasi-increasing function $f$ and a quasidecreasing function $g$. The system $\mathfrak{Q}$ is assumed to be continuously and densely ordered. No such assumptions regarding $B$ are needed. Instead, the definitions of quasi-increasing and quasi-decreasing functions must be slightly modified. For example, a function $f$ on $A$ to $B$ will be called quasi-increasing if, for every non-empty subset $X$ of $A$ and for every $b \in B$ we have

$$
f(U X) \geq b \text { whenever } f(x) \geq b \text { for every } x \in X
$$

and 


$$
f(\cap X) \leq b \text { whenever } f^{\prime}(x) \leq b \text { for every } x \in X
$$

By repeating with small changes the proof of Theorem 3, we see that under these assumptions the conclusions of the theorem remain valid. (The only change which is not obvious is connected with the fact that the system $B$ is not assumed to be densely ordered; therefore we cannot claim the existence of an element $a \in B$ which satisfies (4), and we have to distinguish two cases, dependent on whether an element $a$ with this property exists or not.) Theorem 4 can of course be generalized in the same way.

Theorems 3 and 4 thus generalized can be applied in particular to real functions defined on a closed interval $[a, b]$ of real numbers. In application to real functions Theorem 3 can easily be derived from Theorem 4. In fact, if $f$ is a quasi-increasing real function and $g$ a quasi-decreasing real function on the interval $[a, b]$, then the function $f^{\prime}$ defined by the formula

$$
f^{\prime}(x)=f(x)-g(x)
$$

is clearly quasi-increasing; by applying Theorem 4 to this function, we obtain the conclusions of Theorem 3 for $f$ and $g$. Hence the fixpoint theorem (Theorem 1) for increasing real functions is also a simple consequence of Theorem 4. Finally, since every continuous function is quasi-increasing, and since, in the real domain, continuous functions in our terminology coincide with continuous functions in the usual sense, Theorem 4 is a generalization of the well-known Weierstrass theorem on continuous real functions. ${ }^{4}$ )

Returning to Theorem 3 for simply ordered systems, if we assume that both functions $f$ and $g$ are continuous, we can strengthen the conclusion of the theorem; in fact we can show, not only that the system $\langle P, \leq\rangle$ is continuously

4 Theorem 3 (for both simply ordered systems and real functions) was originally stated under the assumption that the function $f$ is increasing and the function $g$ is continuous; see [3]. In 1949 A. P. Morse noticed that this result in the real domain could be improved; in fact, he obtained Theorem 4 for real functions-under a different, though equivalent, definition of a quasi-increasing function. By his definition, a real function $f$ on an interval $[a, b]$ is quasi-increasing if it is upper semicontinuous on the left and lower semicontinuous on the right, that is, if

$$
\varlimsup_{x \rightarrow d} f(x) \leq f(d) \leq \underset{x \rightarrow d+}{\lim _{x \rightarrow}} f(x) \text { for every } d \in[a, b] .
$$

By generalizing this observation, the author arrived at the present abstract formulations of Theorems 3 and 4. According to a recent remark of Morse, the first part of the conclusion of Theorem 4, that is, the statement that the set $P$ is not empty, holds in the real domain for a still more comprehensive class of functions; in fact, for all real functions which satisfy the condition obtained from (i) by replacing $\underline{\lim }$ by $\overline{\lim }$ on the right side of the double inequality (or else by replacing $\overline{\lim }$ by $\underline{\text { lim }}$ on the left side). 
ordered, but also that, for every nonempty subset $X$ of $P$, the least upper bound of $X$ in $\langle P, \leq\rangle$ coincides with the least upper bound of $X$ in $\langle A, \leq\rangle$, and similarly for the greatest lower bound. In application to real functions this means that the set $P$ of real numbers is, not only continuously ordered, but also closed in the topological sense. Analogous remarks apply to Theorem 4.

\section{Applications to Boolean algebras and the theory of set-theoretical equi-} valence. As is known, a Boolean algebra can be defined as a lattice $\mathfrak{U}=\langle A, \leq\rangle$, with 0 and 1 , in which for every element $b \in A$ there is a uniquely determined element $\bar{b} \in A$ (called the complement of $b$ ), such that

$$
b \cup \bar{b}=1 \text { and } b \cap \bar{b}=0 \text {. }
$$

Given any two elements $a, b \in A$, we shall denote by $a-b$ their difference, that is, the element $a \cap \bar{b}$. If $\mathscr{U}=\langle A, \leq\rangle$ is a Boolean algebra and $a \in A$, then $\mathfrak{X}^{\circ}=\langle[0, a], \leq\rangle$ is also a Boolean algebra, though the complement of an element $b$ in $2{ }^{\prime}$ does not coincide with the complement of $b$ in 2 .

By applying the lattice-theoretical fixpoint theorem we obtain:

\section{THEOREM 5. Let}

(i) $\mathscr{A}=\langle A, \leq\rangle$ be a complete Boolean algebra,

(ii) $a, b$ be any elements of $A, f$ be an increasing function on $[0, a]$ to $A$, and $g$ an increasing function on $[0, b]$ to $A$.

Then there are elements $a^{\prime}, b^{\prime} \in A$ such that

$$
f\left(a-a^{\prime}\right)=b^{\prime} \text { and } g\left(b-b^{\prime}\right)=a^{\prime} .
$$

Proof. Consider the function $h$ defined by the formula

$$
h(x)=f(a-g(b-x)) \text { for every } x \in A \text {. }
$$

Let $x$ and $y$ be any elements in $A$ such that

$$
x \leq y .
$$

We have then

$$
b-x \geq b-y \text {; }
$$

and since $b-x$ and $b-y$ are in $[0, b]$, and $g$ is an increasing function on $[0, b]$ to $A$, we conclude that 


$$
g(b-x) \geq g(b-y)
$$

and

$$
a-g(b-x) \leq a-g(b-y)
$$

Hence, the elements $a-g(b-x)$ and $a-g(b-y)$ being in $[0, a]$, and $f$ being an increasing function on $[0, a]$ to $A$, we obtain

$$
f(a-g(b-x)) \leq f(a-g(b-y)),
$$

that is, by (1),

$$
h(x) \leq h(y)
$$

Thus $h$ is an increasing function on $A$ to $A$, and consequently, by Theorem 1 , it has a fixpoint $b^{\prime}$. Hence, by (1),

$$
f\left(a-g\left(b-b^{\prime}\right)\right)=b^{\prime} .
$$

We put

$$
g\left(b-b^{\prime}\right)=a^{\prime}
$$

From (2) and (3) we see at once that the elements $a^{\prime}$ and $b^{\prime}$ satisfy the conclusion of our theorem.

If in the hypothesis of Theorem 5 we assume in addition that $f(a) \leq b$ and $g(b) \leq a$, we can obviously improve the conclusion by stating that there are elements $a^{\prime}, a^{\prime \prime}, b^{\prime}, b^{\prime \prime} \in A$ for which

$$
\begin{gathered}
a=a^{\prime} \cup a^{\prime \prime}, \quad b=b^{\prime} \cup b^{\prime \prime}, \quad a^{\prime} \cap a^{\prime \prime}=b^{\prime} \cap b^{\prime \prime}=0, \\
f\left(a^{\prime \prime}\right)=b^{\prime} \text { and } g\left(b^{\prime \prime}\right)=a^{\prime} .^{5}
\end{gathered}
$$

Theorem 5 has interesting applications in the discussion of homogeneous elements. Given a Boolean algebra $\mathfrak{U}=\langle A, \leq\rangle$, two elements $a, b \in A$ are called homogeneous, in symbols $a \approx b$, if the Boolean algebras $([0, a], \leq\rangle$ and $\langle[0, b], \leq\rangle$ are isomorphic. In other words, $a \approx b$ if and only if there is a function $f$ satisfying the following conditions: the domain of $f$ is $[0, a]$; the range of $f$ is $[0, b]$; the formulas $x \leq y$ and $f(x) \leq f(y)$ are equivalent for any

5 In this more special form Theorem 5 is a generalization of a set-theoretical theorem obtained by Knaster and the author; see [3]. 
$x, y \in[0, a]$. Various fundamental properties of the homogeneity relation easily follow from this definition; for example, we have:

THEOREM 6. $\mathscr{U}=\langle A, \leq\rangle$ being an arbitrary Boolean algebra,

(i) $a \approx a$ for every $a \in A$;

(ii) if $a, b \in A$ and $a \approx b$, then $b \approx a$;

(iii) if $a, b, c \in A, a \approx b$, and $b \approx c$, then $a \approx c$;

(iv) if $a_{1}, a_{2}, b_{1}, b_{2} \in A, a_{1} \cap a_{2}=0=b_{1} \cap b_{2}$,

$$
a_{1} \approx b_{1} \text {, and } a_{2} \approx b_{2} \text {, then } a_{1} \cup a_{2} \approx b_{1} \cup b_{2} \text {; }
$$

(v) if $a, b_{1}, b_{2} \in A, b_{1} \cap b_{2}=0$, and $a \approx b_{1} \cup b_{2}$,

then there are elements $a_{1}, a_{2} \in A$ such that $a_{1} \cup a_{2}=a_{3}, a_{1} \cap a_{2}=0, a_{1} \approx b_{1}$, and $a_{2} \approx b_{2}$.

In what follows we shall use parts (i)-(iii) of Theorem 6 without referring to them explicitly. If now we restrict our attention to complete Boolean algebras, we can establish various deeper properties of the homogeneity relation by applying Theorem 5. We start with the following:

THEOREM 7. $2 \mathcal{E}=\langle A, \leq\rangle$ being a complete Boolean algebra, if

$$
a, b_{1}, b_{2}, c, d \in A, b_{1} \cap b_{2}=0, c \approx d, \text { and } a \cup c \approx b_{1} \cup b_{2} \cup d \text {, }
$$

then there are elements $a_{1}, a_{2} \in A$ such that

$$
a_{1} \cup a_{2}=a, a_{1} \cap a_{2}=0, a_{1} \cup c \approx b_{1} \cup d \text {, and } a_{2} \cup c \approx b_{2} \cup d \text {. }
$$

Proof. By the definition of homogeneity, the formula $c \approx d$ implies the existence of a function $f$ which maps isomorphically the Boolean algebra $\langle[0, c]$, $\leq\rangle$ onto the Boolean algebra $\langle[0, d]$, $\leq\rangle$; we have in particular

$$
f(c)=d \text {. }
$$

Similarly, the formula $a \cup c \approx b_{1} \cup b_{2} \cup d$ implies the existence of a function $g$ which maps isomorphically $\left\langle\left[0, b_{1} \cup b_{2} \cup d\right], \leq\right\rangle$ onto $\langle[0, a \cup c], \leq\rangle$, and we have

$$
g\left(b_{1} \cup b_{2} \cup d\right)=a \cup c .
$$


We can assume for a while that the domain of $g$ has been restricted to the interval $\left[0, b_{1} \cup d\right]$. Thus, $f$ is an increasing function on $[0, c]$ to $A, g$ is an increasing function on $\left[0, b_{1} \cup d\right]$ to $A$, and by applying Theorem 5 we obtain two elements $c^{\prime}, d^{\prime}$ such that

$$
f\left(c-c^{\prime}\right)=d^{\prime} \text { and } g\left(\left(b_{1} \cup d\right)-d^{\prime}\right)=c^{\prime} \text {. }
$$

The functions $f$ and $g$ being increasing, formulas (1)-(3) imply

$$
d^{\prime} \leq d \text { and } c^{\prime} \leq a \cup c
$$

We now let

$$
a_{1}=c^{\prime}-c \text { and } a_{2}=a-a_{1} \text {. }
$$

By (4) we have $c^{\prime}-c \leq a$, and hence, by (5),

$$
a_{1} \cup a_{2}=a \text { and } a_{1} \cap a_{2}=0
$$

From (4) and (5) we also obtain

$$
d^{\prime} \cup\left[\left(b_{1} \cup d\right)-d^{\prime}\right]=b_{1} \cup d \text { and } d^{\prime} n\left[\left(b_{1} \cup d\right)-d^{\prime}\right]=0 \text {. }
$$

Since $f$ maps isomorphically $\langle[0, c], \leq\rangle$ onto $\langle[0, d], \leq\rangle$, we conclude from (3) that it also maps isomorphically $\left\langle\left[0, c-c^{\prime}\right], \leq\right\rangle$ onto $\left\langle\left[0, d^{\prime}\right], \leq\right\rangle$ and that consequently

$$
c-c^{\prime} \approx d^{\prime}
$$

Analogously, by (3),

$$
c^{\prime} \approx\left(b_{1} \cup d\right)-d^{\prime}
$$

By Theorem 6 (iv), formulas ( 7 ) - (10) imply

$$
a_{1} \cup c \approx b_{1} \cup d \text {. }
$$

Furthermore, from (4) and (5) we derive

(12) $\left(c \cap c^{\prime}\right) \cup\left[(a \cup c)-c^{\prime}\right]=a_{2} \cup c$ and $\left(c \cap c^{\prime}\right) \cap\left[(a \cup c)-c^{\prime}\right]=0$, 


$$
\left(d-d^{\prime}\right) \cup\left[\left(b_{2}-d\right) \cup d^{\prime}\right]=b_{2} \cup d \text { and }\left(d-d^{\prime}\right) \cap\left[\left(b_{2}-d\right) \cup d^{\prime}\right]=0 \text {. }
$$

The function $f$ being an isomorphic transformation, we obtain, with the help of (1) and (3),

$$
f\left(c \cap c^{\prime}\right)=f\left(c-\left(c-c^{\prime}\right)\right)=f(c)-f\left(c-c^{\prime}\right)=d-d^{\prime},
$$

and hence, by arguing as above in the proof of (9),

$$
c \cap c^{\prime} \approx d-d^{\prime} \text {. }
$$

Since, by (4) and the hypothesis,

$$
\left(b_{2}-d\right) \cup d^{\prime}=\left(b_{1} \cup b_{2} \cup d\right)-\left[\left(b_{1} \cup d\right)-d^{\prime}\right],
$$

we conclude analogously, with the help of (2) and (3), that

$$
\left.g\left(b_{2}-d\right) \cup d^{\prime}\right)=g\left(b_{1} \cup b_{2} \cup d\right)-g\left(\left(b_{1} \cup d\right)-d^{\prime}\right)=(a \cup c)-c^{\prime}
$$

and therefore

$$
(a \cup c)-c^{\prime} \approx\left(b_{2}-d\right) \cup d^{\prime} .
$$

From (12)-(15), by applying Theorem 6 (iv) again, we get

$$
a_{2} \cup c \approx b_{2} \cup d \text {. }
$$

By (6), (11), and (16), the proof is complete.

In deriving the remaining theorems of this section we shall apply exclusively those properties of the homogeneity relation which have been established in Theorems 6 and 7; thus the results obtained will apply to every binary relation (between elements of a complete Boolean algebra) for which these two theorems hold. It may be noticed in this connection that Theorem $6(\mathrm{v})$ restricted to complete Boolean algebras is a simple consequence of Theorems 6 (i) and 7.

The orem 8 (MEAN-VAluE ThEOREM). $\mathscr{U}=\langle A, \leq\rangle$ being a complete Boolean algebra, if $a, b, c, a^{\prime}, c^{\prime} \in A, a \leq b \leq c, a^{\prime} \leq c^{\prime}, a \approx a^{\prime}$, and $c \approx c^{\prime}$, then there is an element $b^{\prime} \in A$ such that $a^{\prime} \leq b^{\prime} \leq c^{\prime}$ and $b \approx b^{\prime}$.

Proof. We apply Theorem 7 , with $a, b_{1}, b_{2}, c, d$ respectively replaced by $c^{\prime}-a^{\prime}, b-a, c-b, a^{\prime}, a$, and we conclude that there are elements $a_{1}, a_{2} \in A$ 
such that

$$
c^{\prime}-a^{\prime}=a_{1} \cup a_{2} \text { and }(b-a) \cup a \approx a_{1} \cup a^{\prime}
$$

The element $b^{\prime}=a_{1} \cup a^{\prime}$ clearly satisfies the conclusion of our theorem.

THEOREM 9. $\mathcal{U}=\langle A, \leq\rangle$ being a complete Boolean algebra, for any elements $a, b \in A$ the following two conditions are equivalent:

(i) there is an element $a_{1} \in A$ such that $a \approx a_{1} \leq b$;

(ii) there is an element $b_{1} \in A$ such that $a \leq b_{1} \approx b$.

Proof. To derive (ii) from (i), we consider an arbitrary element $a_{1}$ satisfying (i), and we apply Theorem 8 with $a_{3}, c_{s} a^{\prime}, c^{\prime}$ respectively replaced by $a_{1}$, $1, a, 1$. The implication in the opposite direction follows immediately from Theorem $6(\mathrm{v})$ (and hence holds in an arbitrary Boolean algebra).

THEOREM 10 (EQUIVALENCE THEOREM). $2(=\langle A, \leq\rangle$ being a complete Boolean algebra, if $a, b, c \in A, a \leq b \leq c$, and $a \approx c$, then $a \approx b \approx c$.

Proof. This follows immediately from Theorem 8 with $a^{\prime}=c^{\prime}=c$.

THEOREM 11. $\mathcal{Y}=\langle A, \leq\rangle$ being a complete Boolean algebra, for any elements $a_{1}, a_{2}, b \in A$ the formulas

(i) $a_{1} \cup b \approx a_{2} \cup b \approx b$ and

and

(ii) $a_{1} \cup a_{2} \cup b \approx b$

are equivalent.

Proof. Obviously,

$$
b \leq a_{1} \cup b \leq a_{1} \cup a_{2} \cup b \text { and } b \leq a_{2} \cup b \leq a_{1} \cup a_{2} \cup b
$$

Hence (ii) implies (i) by Theorem 10.

Assume now, conversely, that (i) holds. We clearly have

$$
\left[a_{2}-\left(a_{1} \cup b\right)\right] \cap\left(a_{1} \cup b\right)=\left[a_{2}-\left(a_{1} \cup b\right)\right] \cap b=0
$$

and

$$
a_{2}-\left(a_{1} \cup b\right) \approx a_{2}-\left(a_{1} \cup b\right)
$$

By Theorem 6 (iv), these two formulas together with (i) imply 


$$
a_{1} \cup a_{2} \cup b=\left[a_{2}-\left(a_{1} \cup b\right)\right] \cup\left(a_{1} \cup b\right) \approx\left[a_{2}-\left(a_{1} \cup b\right)\right] \cup b .
$$

Since

$$
\left[a_{2}-\left(a_{1} \cup b\right)\right] \cup b \leq a_{2} \cup b \leq a_{1} \cup a_{2} \cup b \text {, }
$$

we derive from (1), by applying Theorem 10 ,

$$
a_{2} \cup b \approx a_{1} \cup a_{2} \cup b \text {. }
$$

Formulas (i) and (2) obviously imply (ii), and the proof is complete.

Various properties of the relation of homogeneity can conveniently be expressed in terms of another, related relation which is denoted by $\preceq$. Thus $\mathscr{U}=\langle A, \leq\rangle$ being a Boolean algebra, and $a, b$ being any elements of $A$, we write $a \preceq b$ if there is an element $a_{1} \in A$ such that $a \approx a_{1} \leq b$; in case the algebra $\mathscr{U}$ is complete, an equivalent formulation of this condition is given in Theorem 9(ii). Theorems 8 and 10 can now be put in a somewhat simpler, though essentially equivalent, form:

MEAN-VALUE THEOREM. $\mathfrak{U}=\langle A, \leq\rangle$ being a complete Boolean algebra, if $a, b, c \in A, a \leq c$, and $a \preceq b \preceq c$, then there is an element $b^{\prime} \in A$ such that $a \leq b^{\prime} \leq c$ and $b \approx b^{\prime}$.

EQUIVALENCE THEOREM. $\mathscr{U}=\langle A, \leq\rangle$ being a complete Boolean algebra, if $a, b \in A, a \preceq b$, and $b \preceq a$, then $a \approx b$.

We shall give two further results formulated in terms of $\preceq$.

THEOREM 12. $\mathscr{X}=\langle A, \leq\rangle$ being a complete Boolean algebra, if

$$
a_{1}, a_{2}, c_{1}, c_{2} \in A, a_{1} \leq c_{1}, a_{1} \preceq c_{2}, a_{2} \preceq c_{1} \text {, and } a_{2} \leq c_{2} \text {, }
$$

then there are elements $b_{1}, b_{2} \in A$ such that $a_{1} \leq b_{1} \leq c_{1}, a_{2} \leq b_{2} \leq c_{2}$, and $b_{1} \approx b_{2}$.

Proof. The hypothesis implies the existence of two elements $a_{1}^{\prime}, a_{2}^{\prime}$ such that

$$
a_{1} \approx a_{1}^{\prime} \leq c_{2} \text { and } a_{2} \approx a_{2}^{\prime} \leq c_{1} \text {. }
$$

Since, by (1),

$$
a_{1} \cap a_{2}^{\prime} \leq a_{1} \approx a_{1}^{\prime},
$$

we conclude from Theorem 9 that there is an element $d$ for which 


$$
a_{1} \cap a_{2}^{\prime} \approx d \leq a_{1}^{\prime}
$$

We have, by (1),

$$
a_{2} \approx\left(a_{1} \cap a_{2}^{\prime}\right) \cup\left(a_{2}^{\prime}-a_{1}\right) \text { and }\left(a_{1} \cap a_{2}^{\prime}\right) \cap\left(a_{2}^{\prime}-a_{1}\right)=0 ;
$$

hence, by Theorem $6(\mathrm{v})$, there are elements $e_{1}, e_{2}$ such that

$$
\begin{aligned}
& a_{2}=e_{1} \cup e_{2} \text { and } e_{1} \cap e_{2}=0, \\
& e_{1} \approx a_{1} \cap a_{2}^{\prime} \text { and } e_{2} \approx a_{2}^{\prime}-a_{1} .
\end{aligned}
$$

By (1)-(4) and the hypothesis,

$$
d \leq a_{1}^{\prime} \leq c_{2}, e_{1}<c_{2}, d \approx e_{1} \text {, and } c_{2} \approx c_{2} ;
$$

hence, by Theorem 8 , there is an element $f$ for which

$$
e_{1} \leq f \leq c_{2} \text { and } a_{1}^{\prime} \approx f \text {. }
$$

Since, by (4),

$$
e_{2}-f \leq e_{2} \approx a_{2}^{\prime}-a_{1},
$$

Theorem 9 implies the existence of an element $g$ with

$$
e_{2}-f \approx g \leq a_{2}^{\prime}-a_{1} \text {. }
$$

We now put

$$
b_{1}=a_{1} \cup g \text { and } b_{2}=f \cup\left(e_{2}-f\right)=f \cup e_{2} .
$$

By (1), (3), (5), (6), (7), and the hypothesis, we obtain

$$
a_{1} \leq b_{1} \leq c_{1} \text { and } a_{2} \leq b_{2} \leq c_{2} .
$$

By (5) and (6) we have

$$
a_{1} \cap g=f \cap\left(e_{2}-f\right)=0, a_{1} \approx f, g \approx e_{2}-f ;
$$

hence, by (7) and Theorem 6(iv), we get

$$
b_{1} \approx b_{2} \text {. }
$$

From ( 8 ) and (9) we see that the elements $b_{1}$ and $b_{2}$ satisfy the conclusion of our theorem. 
From the theorem just proved, by letting $a_{1}=c_{1}$, we derive as an immediate consequence the mean-value theorem; if we put $a_{1}=c_{1}$ and $a_{2}=c_{2}$, we obtain the equivalence theorem. A further consequence of Theorem 12 is:

TheOrem 13 (INTER POLATION THEOREM). $\mathfrak{A}=\langle A, \leq\rangle$ being a complete Boolean algebra, if $a_{1}, a_{2}, c_{1}, c_{2} \in A$ and $a_{i} \preceq c_{j}$ for $i_{9} j=1,2$, then there is an element $b \in A$ such that $a_{i} \preceq b \preceq c_{j}$ for $i, j=1,2$.

Proof. The hypothesis implies the existence of two elements $a_{1}^{\prime}$ and $a_{2}^{\prime}$ for which

$$
a_{1} \approx a_{1}^{\prime} \leq c_{1} \text { and } a_{2} \approx a_{2}^{\prime} \leq c_{2} \text {. }
$$

Hence, as is easily seen,

$$
a_{1}^{\prime} \leq c_{1}, a_{1}^{\prime} \preceq c_{2}, a_{2}^{\prime} \preceq c_{1}, a_{2}^{\prime} \leq c_{2} .
$$

Consequently, by Theorem 12, there are elements $b_{1}, b_{2}$ such that

$$
a_{1}^{\prime} \leq b_{1} \leq c_{1}, a_{2}^{\prime} \leq b_{2} \leq c_{2} \text {, and } b_{1} \approx b_{2} .
$$

From (1) and (2), with the help of Theorem 9, we obtain

$$
a_{i} \preceq b_{1} \preceq c_{j} \quad \text { for } \quad i_{9} j=1,2 .
$$

Thus the element $b=b_{1}$ satisfies the conclusion of our theorem.

From Theorems 7 and $11-13$ we obtain by induction more general results in which the couples $\left\langle a_{1}, a_{2}\right\rangle,\left\langle b_{1}, b_{2}\right\rangle\left\langle c_{1}, c_{2}\right\rangle$ are replaced by finite sequences

$$
\left\langle a_{1}, \cdots, a_{n}\right\rangle,\left\langle b_{1}, \cdots, b_{n}\right\rangle,\left\langle c_{1}, \cdots, c_{n}\right\rangle
$$

with an arbitrary number $n$ of terms; in Theorem 13 the couples $\left\langle a_{1}, a_{2}\right\rangle$ and $\left\langle c_{1}, c_{2}\right\rangle$ can be replaced by two finite sequences with different numbers of terms. The results discussed can be further extended to infinite sequences; however, these extensions seem to require a different method of proof, and we see no way of deriving them by means of elementary arguments from the fixpoint theorem of $\$ 1 .^{6}$

${ }^{6}$ Theorems 6-13 concerning the relation of homogeneity and their applications to cardinal products of Boolean algebras and to the theory of set-theoretical equivalence are not essentially new. (Theorem 12 is new, but it can be regarded simply as a new formulation of the interpolation the orem 13.) All these results are stated explicitly or implicitly in $[7, \delta \& 11,12,15-17]$, where historical references to earlier publications can also be found. However, the method applied in [7] is different from that in the present paper and is not directly related to any fixpoint theorem. Also, the axiom of choice is not involved at all in the present discussion, while the situation in [7] is in this respect more complicated (compare, for instance, the remarks starting on page 239). 
All the results of this section, except Theorem 5, remain valid if the Boolean algebra $\mathscr{U}=\langle A, \leq\rangle$ is assumed to be not necessarily complete, but only countably-complete ( $\sigma$-complete). This can be seen in the following way. To prove Theorem 5 we have constructed, in terms of two given increasing functions $f$ and $g$, a new function $h$, and we have shown that this function $h$ is increasing and hence has a fixpoint. In the subsequent discussion, Theorem 5 has been applied only once, namely in the proof of Theorem 7. The functions $f$ and $g$ involved in this application not only are increasing, but have much stronger properties, in fact, the distributive properties under countable joins and meets; that is, for every infinite sequence $\left\langle a_{1}, \ldots, a_{n}, \ldots\right\rangle$ we have

$$
\begin{aligned}
& f\left(a_{1} \cup \cdots \cup a_{n} \cup \cdots\right)=f\left(a_{1}\right) \cup \cdots \cup f\left(a_{n}\right) \cup \cdots, \\
& f\left(a_{1} \cap \ldots \cap a_{n} \cap \cdots\right)=f\left(a_{1}\right) \cap \cdots \cap f\left(a_{n}\right) \cap \cdots,
\end{aligned}
$$

and similarly for $g$. It can be shown that the function $h$ constructed from $f$ and $g$ in the way indicated in the proof of Theorem 5 also has these distributive properties. It is also easily seen that, in any countably-complete Boolean algebra (and, more generally, in any countably-complete lattice with 0 ), every function $h$ which is distributive under countable joins has at least one fixpoint $a$; in fact,

$$
a=0 \cup h(0) \cup h(h(0)) \cup \cdots
$$

The results obtained in this section have interesting consequences concerning isomorphism of cardinal (direct) products of Boolean algebras. To obtain these consequences it suffices to notice that every system of Boolean algebras $\left\langle\mathfrak{U}_{i}\right\rangle$ can be represented by means of a system of disjoint elements $\left\langle a_{i}\right\rangle$ of a single Boolean algebra $\mathfrak{L}$ (in fact, of the cardinal product of all algebras $\mathfrak{U}_{i}$ ) in such a way that $(i)$ each algebra $\mathfrak{U}_{i}$ is isomorphic to the subalgebra $\left\langle\left[0, a_{i}\right], \leq\right\rangle$ of $\mathfrak{2}$; hence (ii) two algebras $\mathfrak{A}_{i}$ and $\mathfrak{L}_{j}$ are isomorphic $\left(\mathfrak{A}_{i} \cong \mathfrak{2}_{j}\right)$ if and only if the elements $a_{i}$ and $a_{j}$ are homogeneous $\left(a_{i} \approx a_{j}\right)$; (iii) for $i \neq j$, we have $\mathfrak{A}_{i} \times \mathfrak{Z}_{j}=\mathfrak{A}_{k}$ if and only if $a_{i} \cup a_{j} \approx a_{k}$; (iv) $\mathfrak{L}_{i}$ is isomorphic to a factor of $\mathfrak{A}_{k}$ if and only if $a_{i} \preceq a_{k}$. Keeping this in mind, we derive, for example, the following corollary from Theorem 11:

$\mathfrak{Z}_{1}, \mathfrak{U}_{2}, \mathbb{B}$ being three complete Boolean algebras, we have

$$
\mathcal{H}_{1} \times B \cong \mathcal{H}_{2} \times B \cong B
$$

if and only if

$$
\mathfrak{U}_{1} \times \mathfrak{U}_{2} \times \mathfrak{B} \cong \mathfrak{B} \text {. }
$$


Results of this type can again be extended to countably-complete Boolean algebras.

Any given sets $A, B, C, \cdots$ can be regarded as elements of a complete Boolean algebra; in fact, of the algebra formed by all subsets of the union $A \cup B \cup C \cup \cdots$, with set-theoretical inclusion as the fundamental relation. As is easily seen, two sets $A$ and $B$ treated this way are homogeneous in the Boolean-algebraic sense if and only if they are set-theoretically equivalent, that is, have the same power. Hence, as particular cases of theorems on homogeneous elements, we obtain various results concerning set-theoretical equivalence; for instance, Theorem 10 yields the well-known Cantor-Bernstein theorem. ${ }^{7}$

4. Applications to topology. ${ }^{8}$ By a derivative algebra we understand a system $\mathfrak{U}=\langle A, \leq, D\rangle$ in which $\langle A, \leq\rangle$ is a Boolean algebra and $D$ is a unary operation (function) on $A$ to $A$ assumed to satisfy certain simple postulates; the main consequence of these postulates which is involved in our further discussion is the fact that $D$ is increasing. The element $D_{x}$ (for any given $x \in A$ ) is referred to as the derivative of $x$. The derivative algebra $2 \mathcal{C}$ is called complete if the Boolean algebra $\langle A, \leq\rangle$ is complete.

In topology the notion of the derivative of a set is either treated as a fundamental notion in terms of which the notion of a topological space is characterized, or else it is defined in terms of other fundamental notions (for example, the derivative of a point set $X$ is defined as the set of all limit points of $X$ ). At any rate, all point sets of a topological space form a complete derivative algebra under the set-theoretical relation of inclusion and the topological operation of derivative. Hence the theorems on complete derivative algebras can be applied to arbitrary topological spaces.

$\mathfrak{U}=\langle A, \leq, \mathrm{D}\rangle$ being a derivative algebra, an element $a \in A$ is called closed if $\mathrm{D} a \leq a$; it is called dense-in-itself if $\mathrm{D} a \geq a$, and perfect if $\mathrm{D} a=a$; it is called $s$ cattered if there is no element $x \leq a$ different from 0 which is dense-initself.

As a consequence of the fixpoint theorem we obtain:

\section{Theorem 14 (Generalized Cantor-Bendixon theorem).}

7 These extensions can be found in [7]. The proof of Theorems 12 and 13 extended to infinite sequences requires an application of the axiom of choice (to denumerable families of sets ). Compare the preceding footnote.

8 In connection with this section see [4, pp. $182 \mathrm{f}$.$] ; compare also [5], in particular$ pp. $38 \mathrm{f}$. and 44 . 


$$
\mathscr{W}=\langle A, \leq, \mathrm{D}\rangle
$$

being a complete derivative algebra, every closed element $a \in A$ has a decomposition

$$
a=b \cup c, b \cap c=0,
$$

where the element $b \in A$ is perfect and the element $c \in A$ is scattered.

Proof. We put

$$
b=\mathrm{UE}_{x}[a \cap \mathrm{D} x \geq x] \text { and } c=a-b .
$$

Hence obviously

$$
a=b \cup c \text { and } b \cap c=0 \text {. }
$$

$D$ being an increasing function on $A$ to $A$, the same clearly applies to the function $\mathrm{D}_{a}$ defined by the formula

$$
\mathrm{D}_{a} x=a \cap \mathrm{D}_{x} \text { for every } x \in A \text {. }
$$

Hence, by Theorem 1 , we conclude from ( 1 ) that $b$ is a fixpoint of $D_{a}$; that is,

$$
b=\mathrm{D}_{a} b=a \cap \mathrm{D} b .
$$

Consequently $b \leq a$ and $\mathrm{D} b \leq \mathrm{D} a$; since the element $a$ is closed, we have $\mathrm{D} a \leq a, \mathrm{D} b \leq a$ and therefore, by (3), $b=\mathrm{D} b$; that is, the element $b$ is perfect. If an element $x \leq c$ is dense-in-itself, that is, $D x \geq x$, we have, by ( 1 ),

$$
a \cap D_{x} \geq x,
$$

and hence $x \leq b$; therefore, by (2), $x=0$. Thus the element $c$ is scattered. This completes the proof.

It should be mentioned that the operation $D$ in a derivative algebra

$$
\mathfrak{U}=\langle A, \leq, D\rangle
$$

is assumed to be not only increasing, but distributive under finite joins, that is,

$$
\mathrm{D}(x \cup y)=\mathrm{D} x \cup \mathrm{D} y \text { for any } x, y \in A \text {. }
$$

Under this assumption we can improve Theorem 14 by showing that every closed 
element $a \in A$ has a unique decomposition

$$
a=b \cup c, b \cap c=0 \text {, }
$$

where $b$ is perfect and $c$ is scattered. In fact, let

$$
a=b^{\prime} \cup c^{\prime}, b^{\prime} \cap c^{\prime}=0
$$

be another decomposition of this kind. We then have

$$
b=\mathrm{D} b \leq \mathrm{D}\left(b \cup b^{\prime}\right)=\mathrm{D}\left(\left(b-b^{\prime}\right) \cup b^{\prime}\right)=\mathrm{D}\left(b-b^{\prime}\right) \cup \mathrm{D} b^{\prime}=\mathrm{D}\left(b-b^{\prime}\right) \cup b^{\prime} .
$$

Hence

$$
b-b^{\prime} \leq \mathrm{D}\left(b-b^{\prime}\right)
$$

that is, $b-b^{\prime}$ is dense-in-itself. Since, moreover, $b-b^{\prime} \leq c^{\prime}$, and $c^{\prime}$ is scattered, we conclude that $b-b^{\prime}=0$. Similarly we get $b^{\prime}-b=0$. Consequently $b=b^{\prime}$, and hence also $c=c^{\prime}$.

If, instead of Theorem 1, we apply Theorem 5, we obtain the following result (of which, however, no interesting topological consequences are known):

THEOREM 15. $\mathscr{C}=\langle A, \leq, \mathrm{D}\rangle$ being a complete derivative algebra, every closed element $a \in A$ has two decompositions

$$
a=b \cup c=b^{\prime} \cup c^{\prime}, \quad b \cap c=b^{\prime} \cap c^{\prime}=0,
$$

where $b, c, b^{\prime}, c^{\prime}$ are elements of 'A such that

$$
\mathrm{D}^{\prime}=b \text { and } \mathrm{D}_{c^{\prime}}=c .
$$

Proof. From Theorem 5 (with $a=b$ ) we conclude that there are two elements $c, b^{\prime} \in A$ such that

$$
\mathrm{D}(a-c)=b^{\prime} \text { and } \mathrm{D}\left(a-b^{\prime}\right)=c \text {. }
$$

By putting,

$$
b=a-c \text { and } c^{\prime}=a-b^{\prime}
$$

we obtain, from (1),

$$
\mathrm{D} b=b^{\prime} \text { and } \mathrm{D}_{c^{\prime}}=c .
$$


Since the function $D$ is increasing and the element $a$ is closed, (1) implies

$$
c \leq \mathrm{D} a \leq a \text { and } b^{\prime} \leq \mathrm{D} a \leq a
$$

hence, by (2),

$$
a=b \cup c=b^{\prime} \cup c^{\prime} \text { and } b \cap c=b^{\prime} \cap c^{\prime}=0 \text {. }
$$

By (3) and (4) the proof has been completed.

Theorems 1 and 5 can be applied not only to the operation $\mathrm{D}$, but also to other topological operations which are defined in terms of $D$ and, like the latter, are increasing; for instance, to the operation I defined by the formula

$$
I_{x}=x-D \bar{x}
$$

I $x$ referred to as the interior of the element $x$. Theorem 5 can of course be applied to two different topological operations, provided both are increasing.

\section{REFERENCES}

1. Garrett Birkhoff, Lattice theory, revised edition, Amer. Math. Soc. Colloq. Publ. vol. 25, New York, 1948.

2. Anne C. Davis, A characterization of complete lattices, Pacific J. Math. 5 (1955), $311-319$.

3. B. Knaster, Un théorème sur les fonctions d'ensembles, Ann. Soc. Polon. Math. 6 (1928), 133 - 134.

4. J. C. C. McKinsey and Alfred Tarski, The algebra of topology, Ann. of Math. 45 (1944), $141-191$.

5. Wacław, Sierpiński, Introduction to general topology, Toronto, 1934.

6. Alfred Tarski, A fixpoint theorem for lattices and its applications (preliminary report ), Bull. Amer. Math. Soc. 55 (1949), $1051-1052$ and 1192.

7. - Cardinal algebras, New York, 1949.

\section{UNIVERSITY OF CALIFORNIA, BERKELEY}





\section{PACIFIC JOURNAL OF MATHEMATICS}

\section{EDITORS}

\author{
H. L. ROYDEN \\ Stanford University \\ Stanford, California \\ E. Hewitt \\ University of Washington \\ Seattle 5 , Washington
}

R. P. Dilworth

California Institute of Technology Pasadena 4, California

A. HorN*

University of California

Los Angeles 24, California

\section{ASSOCIATE EDITORS}

\author{
H. BUSEMANN \\ HERBERT FEDERER \\ MARSHALL HALL
}

\author{
P. R. HALMOS \\ HEINZ HOPF
}

ALFRED HORN
R. D. JAMES

BORGE JESSEN

PAUL LÉVY
GEORGE PÓLYA

J. J. STOKER

KOSAKU YOSIDA

\section{SPONSORS}

UNIVERSITY OF BRITISH COLUMBIA

CALIFORNIA INSTITUTE OF TECHNOLOGY

UNIVERSITY OF CALIFORNIA, BERKELEY

UNIVERSITY OF CALIFORNIA, DAVIS

UNIVERSITY OF CALIFORNIA, LOS ANGELES

UNIVERSITY OF CALIFORNIA, SANTA BARBARA

MONTANA STATE UNIVERSITY

UNIVERSITY OF NEVADA

OREGON STATE COLLEGE

UNIVERSITY OF OREGON

UNIVERSITY OF SOUTHERN CALIFORNIA

\author{
STANFORD RESEARCH INSTITUTE \\ STANFORD UNIVERSITY \\ UNIVERSITY OF UTAH \\ WASHINGTON STATE COLLEGE \\ UNIVERSITY OF WASHINGTON
}

AMERICAN MATHEMATICAL SOCIETY HUGHES AIRCRAFT COMPANY SHELL DEVELOPMENT COMPANY

Mathematical papers intended for publication in the Pacific Journal of Mathematics should be typewritten (double spaced), and the author should keep a complete copy. Manuscripts may be sent to any of the editors. Manuscripts intended for the outgoing editors should be sent to their successors. All other communications to the editors should be addressed to the managing editor, Alfred Horn at the University of California, Los Angeles 24, California.

50 reprints of each article are furnished free of charge; additional copies may be obtained at cost in multiples of 50 .

The Pacific Journal of Mathematics is published quarterly, in March, June, September, and December. The price per volume (4 numbers) is $\$ 12.00$; single issues, $\$ 3.50$. Back numbers are available. Special price to individual faculty members of supporting institutions and to individual members of the American Mathematical Society: $\$ 4.00$ per volume; single issues, $\$ 1.25$.

Subscriptions, orders for back numbers, and changes of address should be sent to Pacific Journal of Mathematics, c/o University of California Press, Berkeley 4, California.

Printed at Kokusai Bunken Insatsusha (International Academic Printing Co., Ltd.), No. 10, 1-chome, Fujimi-cho, Chiyoda-ku, Tokyo, Japan.

* During the absence of E. G. Straus.

PUBLISHED BY PACIFIC JOURNAL OF MATHEMATICS, A NON-PROFIT CORPORATION COPYRIGHT 1955 BY PACIFIC JOURNAL OF MATHEMATICS 


\section{Pacific Journal of Mathematics}

\section{Vol. 5, No. $2 \quad$ October, 1955}

Leonard M. Blumenthal, An extension of a theorem of Jordan and von

Neumann ........................................ 161

L. Carlitz, Note on the multiplication formulas for the Jacobi elliptic functions.......................................... 169

L. Carlitz, The number of solutions of certain types of equations in a finite

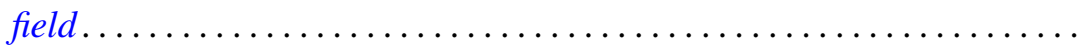

George Bernard Dantzig, Alexander Orden and Philip Wolfe, The generalized simplex method for minimizing a linear form under linear

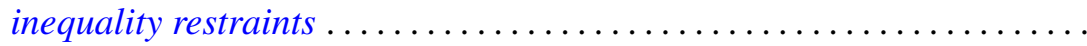

Arthur Pentland Dempster and Seymour Schuster, Constructions for poles and polars in n-dimensions . . . . . . . . . . . . . . . . . . . . 197

Franklin Haimo, Power-type endomorphisms of some class 2 groups ...... 201

Lloyd Kenneth Jackson, On generalized subharmonic functions ......... 215

Samuel Karlin, On the renewal equation ...................... 229

Frank R. Olson, Some determinants involving Bernoulli and Euler numbers of higher order................................ 259

R. S. Phillips, The adjoint semi-group ........................ 269

Alfred Tarski, A lattice-theoretical fixpoint theorem and its applications ... 285

Anne C. Davis, A characterization of complete lattices .............. 311 